\title{
Mitral regurgitation after anthracycline treatment for childhood malignancy
}

\author{
J Allen, J D R Thomson, I J Lewis, J L Gibbs
}

\begin{abstract}
Objective-To investigate the new onset of mitral regurgitation in patients with otherwise normal echocardiograms after anthracycline treatment and to assess its relation to other selected indicators of myocardial damage.

Design-Prospective echocardiographic and electrocardiographic study.

Setting-Tertiary paediatric cardiac referral centre.

Patients-305 patients, aged 2-33 years (median 14 years), treated with cumulative anthracycline doses of between $150-450 \mathrm{mg} / \mathrm{m}^{2}$ (median $180 \mathrm{mg} / \mathrm{m}^{2}$ ) for childhood malignancy. Main outcome measures-Colour flow Doppler detection of mitral regurgitation and its relation to changes in echocardiographic indices of left ventricular function (systolic and diastolic dimensions, fractional shortening) and to changes in the 12 lead ECG; and the prevalence of mitral regurgitation in the anthracycline treated patients in comparison with previously studied normal volunteers of similar age.

Results-34 patients (11.6\%) developed ultrasound detectable mitral regurgitation, which was not apparent clinically, during or after anthracycline treatment, compared with only $1.8 \%$ of a normal population of similar age $(\mathrm{p}<0.0001)$. Nine of the 34 also developed non-specific $\mathrm{T}$ wave abnormalities. All 34 patients had normal systolic function at the time of initial detection of mitral regurgitation, but four later developed impaired left ventricular function $(5,11,20$, and 27 months after the first detection of mitral regurgitation).

Conclusions-Mitral regurgitation occurs much more often in patients treated with anthracyclines than in the normal population. Echocardiographic detection of new mitral regurgitation with or without ECG abnormalities may be an early predictor of anthracycline cardiomyopathy.

(Heart 2001;85:430-432)
\end{abstract}

Keywords: anthracyclines; mitral regurgitation; cardiomyopathy

No easily repeatable, non-invasive test appears to offer early, sensitive, and specific prediction of irreversible anthracycline induced myocardial damage. During our routine echocardiographic follow up of patients treated with anthracyclines, trivial mitral regurgitation was noted anecdotally as a new finding in several cases, in whom fractional shortening remained within normal limits. This finding-along with our previous report that mitral regurgitation in normal children and adolescents is rare prompted the present study. Our aim was to assess the incidence of new onset mitral regurgitation after treatment with anthracyclines, to investigate its relation to other selected indicators of myocardial damage, and to compare the prevalence of mitral regurgitation in these patients with a normal population of similar age.

\section{Methods}

We studied 305 patients (180 male, 125 female) following treatment with anthracycline chemotherapy for childhood malignancy. They were aged between 2 and 31 years (median 14 years). All patients were assessed before starting chemotherapy. Patients who were on active treatment were assessed before each course of treatment if the dose of anthracycline was more than $300 \mathrm{mg} / \mathrm{m}^{2}$, or before alternate courses if the dose was less than $300 \mathrm{mg} / \mathrm{m}^{2}$. On follow up, these patients had assessments after completion of each chemotherapy regimen or earlier if there was any deterioration in their clinical status. In the long term, patients were seen every three years before puberty and thereafter every five years.

At each visit a 12 lead ECG and cross sectional, $M$ mode, and colour flow Doppler echocardiographic examinations were performed by experienced ultrasonographers and the results analysed by a paediatric cardiologist. The ultrasound study was performed in the left lateral position, with a single lead ECG recorded simultaneously. Sedation was not used. A Hewlett-Packard Sonos 1500 phased array ultrasound imaging system (Hewlett Packard Inc, Andover, Massachusetts, USA) was used, and hard copy was taken onto videotape and thermal imaging paper. The highest frequency transducer $(7.5 / 5.5 \mathrm{MHz}, 3.5 /$ $2.7 \mathrm{MHz}$, or $2.5 / 2.0 \mathrm{MHz}$ ) which gave adequate penetration was used. Cross sectional imaging was performed using standard planes. $M$ mode recordings were taken at the level of the tips of the mitral valve perpendicular to the interventricular septum and left ventricular posterior wall. Left ventricular dimensions were measured from leading edge to edge. Left ventricular internal dimensions at end diastole were measured at the onset of the QRS complex, and internal dimensions at end systole were measured at the point of peak posterior motion of the interventricular septum, 

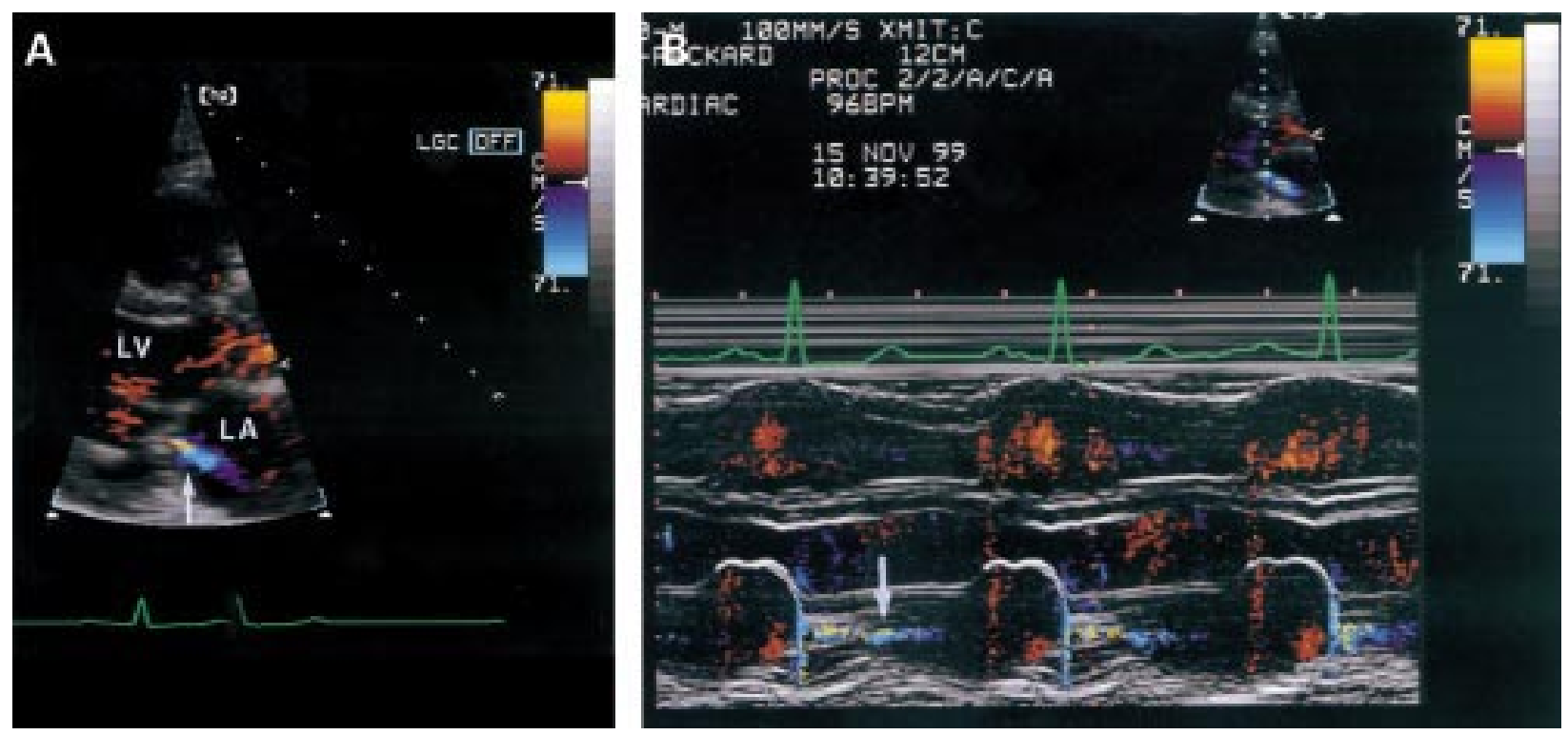

Figure 1 (A) Long axis parasternal echocardiogram showing a jet (arrowed) of trivial mitral regurgitation after anthracycline treatment. (B) Colour M mode recording showing the jet (arrowed) to be pansystolic.

recorded at $100 \mathrm{~mm} / \mathrm{s}$ paper speed. The colour flow wall filter was set at $0.21 \mathrm{~m} / \mathrm{s}$, with a colour width of $30^{\circ}$ and frame rate of $12-14$ frames/second.

New onset mitral regurgitation was defined as a high velocity colour jet detectable after mitral valve closure (fig 1), occurring in a patient with a previously normal echocardiogram. Patients with mitral regurgitation secondary to established cardiomyopathy were not included in the analysis.

\section{Results}

No patients had pre-existing mitral regurgitation on baseline echocardiography. Thirty four patients $(11.6 \%)$ developed new trivial mitral regurgitation, which was not apparent clinically, without any detectable impairment of left ventricular systolic function. Nineteen of these were male and 15 female, and they were aged between 3-27 years (median 14 years) at the time of onset of mitral regurgitation. Cumulative anthracycline dosage received was calculated from chemotherapy protocols, and the maximum ranged from $150-450 \mathrm{mg} / \mathrm{m}^{2}$ (median $225 \mathrm{mg} / \mathrm{m}^{2}$ ). The time from first starting treatment to detection of mitral regurgitation was between $0.3-21$ years (median 3 years).

An additional 12 of the 305 patients (3.9\%) developed mitral regurgitation after left ventricular function had become impaired. These were considered to have functional mitral regurgitation and were therefore not included in the analysis.

Of the 34 patients with normal left ventricular function who developed mitral regurgitation, nine $(26 \%)$ also had non-specific $\mathrm{T}$ wave flattening or inversion. Five had these ECG changes before detection of mitral regurgitation, three developed ECG changes and mitral regurgitation at the same time, and one developed ECG changes 13 months after the onset of mitral regurgitation. Of the nine patients with ECG changes and mitral regurgitation, the ECG returned to normal in three but the mitral regurgitation has persisted in all 34 patients.

Four patients - aged 3, 3, 10, and 16 years at time of first detection of mitral regurgitationdeveloped impaired left ventricular function (fractional shortening $21 \%, 20 \%, 24 \%$, and $18 \%$, respectively) $5,27,11$, and 20 months later, between four months and five years after their first course of anthracycline (total cumulative dose $229,375,140$, and $175 \mathrm{mg} / \mathrm{m}^{2}$ ).

\section{Discussion}

The anthracyclines daunorubicin and doxorubicin are well known to be cardiotoxic. ${ }^{2}$ By the time significant impairment of left ventricular systolic function is detected, myocardial damage is usually irreversible and often progresses even after cessation of chemotherapy. Early detection of cardiotoxicity is clearly desirable, particularly during the course of chemotherapy, when adjustments to the treatment protocol might avoid the development of dilated cardiomyopathy. The major factor determining the risk of developing cardiomyopathy is the total cumulative dose of anthracycline received, with a rapid increase in toxicity with amounts above $550 \mathrm{mg} / \mathrm{m}^{2}{ }^{3}$ Although most current anthracycline protocols use a cumulative dosage of less than $450 \mathrm{mg} / \mathrm{m}^{2}$, cardiomyopathy remains a major complication of treatment.

Comparison of serial ECGs measuring QRS voltage, ST segment and $\mathrm{T}$ wave changes, ${ }^{4-6}$ QT interval, ${ }^{78}$ and heart rhythm ${ }^{9}$ has been used for many years to detect cardiotoxicity, but none of these variables - either singly or in combination-is sensitive or specific in the early prediction of cardiomyopathy.

The most widely used measure of cardiac function in children who have received anthracyclines is cross sectional and $M$ mode echocardiography. Serial increases in left ventricular dimensions or a decrease in fractional shortening may indicate myocardial damage, ${ }^{10}$ 
but may be insensitive to subtle changes in myocardial function and limited in its ability to predict progression and functional outcome. Indices of left ventricular diastolic function, such as the pattern of mitral inflow velocities, have also been reported to be abnormal in some patients after anthracycline treatment, but these changes may be difficult to interpret in individual subjects. ${ }^{11}$ Serial endomyocardial biopsy is probably the most reliable predictor of the extent and potential rate of progression of damage for any individual, ${ }^{12}$ but repeated biopsies carry appreciable risk and require considerable resources, so this approach has not been widely adopted. Similarly, although there is some evidence that serial radionuclide angiography may detect early changes in myocardial function, ${ }^{13}$ the technique has practical difficulties in children and has not found favour in routine practice.

Mitral regurgitation is seen in a proportion of normal adults and is thought to be related to aging. ${ }^{14}$ In a prospective study of normal children and adolescents, we found that mitral regurgitation (defined in identical terms to the present study) was detectable in only $1.8 \%$ of 329 normal volunteers, ${ }^{1}$ in striking contrast ( $p<0.0001$ using the $\chi^{2}$ test) to the $11.6 \%$ incidence in anthracycline treated patients. We found mitral regurgitation in four of 305 patients $(1.3 \%)$ before the start of chemotherapy, suggesting that in children with malignant disease who have not been treated with anthracyclines it is also very uncommon.

Non-specific $T$ wave changes on the ECG occurred more often in patients with new mitral regurgitation than in those without (nine of 34 patients (26\%) and 27 of $259(10.4 \%)$, respectively; $\mathrm{p}=0.016$ using Yates's corrected $\chi^{2}$ test).

Although it is accepted that mitral regurgitation is part of the aging process in adults, the exact mechanism responsible is unclear, and there is no specific evidence implicating the cusps, chordae, or papillary muscles. A minor degree of papillary muscle dysfunction related to a generalised anthracycline induced insult to the myocardium (thought to be mediated by free radicals ${ }^{15}$ ), too subtle to be detected by changes in fractional shortening, seems a reasonable explanation of the trivial degrees of mitral regurgitation seen in our patients. Four of the 34 patients with new onset mitral regurgitation later developed impaired left ventricular function $(5,11,20$, and 27 months after the mitral regurgitation was first detected). Further long term follow up studies will be needed to evaluate the implications of these findings fully, but our preliminary results suggest that an isolated finding of new onset mitral regurgitation may be an early indicator of anthracycline cardiotoxicity.

1 Thomson JDR, Allen J, Gibbs JL. Left heart valve regurgitation in normal children and adolescents. Heart 2000;83:185-7.

2 Bristow MR, Billingham ME, Mason JW, et al. Clinical spectrum of anthracycline antibiotic cardiotoxicity. Cancer Treat Rep 1978;62:873-9.

3 Von Hoff DD, Layard MW, Basa P, et al. Risk factors for doxorubicin-induced congestive heart failure. Ann Intern Med 1979;91:710-12.

4 VonHoff DD, Rozencweig M, Peccart M. The cardiotoxicity of anticancer agents. Semin Oncol 1982;9:23-6.

5 Minow RA. Adriamycin cardiomyopathy; an overview with determination of risk factors Cancer Chemother Rep 1975;6: 195.

6 Weaver SK, Fulkerson PK, Lewis RP, et al. A paucity of chronic electrocardiographic changes with Adriamycin administration. F Electrocardiogr 1978;11:233-6.

7 Schwartz M, Truesdell S. Corrected QT interval prolongation in anthracycline-treated survivors of childhood cancer. f Clin Oncol 1993;11:1906-10.

8 Bender KS, Shematek JP, Leventhal BG, et al. QT interval prolongation associated with anthracycline cardiotoxicity. $\mathcal{F}$ Pediatr 1984; 105:442-4.

9 Larsen RL, Jakacki RI, Vetter VL, et al. Electrocardiographic changes and arrhythmias after cancer therapy in children and young adults. Am F Cardiol 1992;70:73-5.

10 Fortuin NJ, Pawsey CGK. The evaluation of left ventricular function by echocardiography. Am Heart f 1977 93:33-6.

11 Bu'Lock FA, Mott MG, Oakhill A, et al. Left ventricular diastolic function after anthracycline chemotherapy in childhood: relation with systolic function, symptoms and pathophysiology. Br Heart $\mathcal{f} 1995 ; 73$ 340-50.

12 Billingham ME, Bristow MR. Evaluation of anthracycline cardiotoxicity: predictive ability and functional correlation of endomyocardial biopsy. Cancer Treat Symp 1984;3:716.

13 Druck MN, Gulenchyn KY, Evans WK, et al. Radionuclide angiography and endomyocardial biopsy in the assessment of doxorubicin cardiotoxicity. Cancer 1984;53:1667-9.

14 Akasaka T, Yosikawa J, Yoshida K, et al. Age-related valvular regurgitation; a study by pulsed Doppler echocardiography. Circulation 1987;76:262-5.

15 Keizer HG, Pinedo HM, Schuuruis GJ, et al. Doxorubicin (Adriamycin): a critical review of free radical-dependent mechanism of cytotoxicity. Pharmacol Ther 1990;47:219- 